\title{
Mxene-Assisted Organic Electrochemical Transistor Biosensor With Multiple Spiral Interdigitated Electrodes for Sensitive Determination of fPSA/tPSA.
}

\section{Yi-Cheng Zhu}

Shanghai University of Medicine and Health Sciences

\section{Biao Cai}

Shanghai Jiao Tong University School of Medicine Affiliated Renji Hospital

\section{Quan Jiang}

Shanghai University of Medicine and Health Sciences

\section{Yuan Zhang}

Shanghai University of Medicine and Health Sciences

Jianjun Sha ( $\square$ jianjunsha2011@163.com)

Shanghai Jiao Tong University School of Medicine Affiliated Renji Hospital https://orcid.org/00000002-3978-0193

\section{Shaowei Xie}

Shanghai Jiao Tong University School of Medicine Affiliated Renji Hospital

\section{Research Article}

Keywords: prostate specific antigen, organic electrochemical transistor, multiplexed spiral, biomarker

Posted Date: August 4th, 2021

DOI: https://doi.org/10.21203/rs.3.rs-750812/v1

License: (c) (i) This work is licensed under a Creative Commons Attribution 4.0 International License. Read Full License 


\section{Abstract}

Background: The ratio of fPSA/tPSA in the "grey zone" of tPSA with the concentration range between 4 $\mathrm{ng} / \mathrm{ml}$ and $10 \mathrm{ng} / \mathrm{ml}$ is significant for diagnosis of prostate cancer, and highly efficiency quantification of the ratio of fPSA/tPSA remain elusive mainly because of their extremely low concentration in patients' peripheral blood with high biosample complexity.

Methods: We presented an interdigitated spiral-based MXene-assisted organic electrochemical transistors (isMOECTs) biosensor for highly sensitive determination of fPSA/tPSA. The combination of MXene and the interdigitated multiple spiral architecture synergistically assisted the amplification of amperometric signal of biosensor with dual functionalizations of anti-tPSA and anti-fPSA.

Results: The ultrasensitivity of the biosensor was enhanced by tunable multiple spiral architecture and MXene nanomaterials; and the sensor demonstrated enhanced detection limit of tPSA and FPSA down to $0.01 \mathrm{pg} / \mathrm{ml}$, as well as satisfactory selectivity, reproducibility and reliability, which was comparable to the best previous literatures; Moreover, the isMOECTs displayed area under the curve (AUC) value of 0.8138 , confirming the potential applications of isMOECTs in clinics.

Conclusions: The merits of isMOECTs biosensor demonstrated the reliability of MXene-assisted organic electrochemical transistor biosensor with multiple interdigitated spiral for ultrasensitive quantification of fPSA/tPSA, suggesting potential current and future point-of-care testing applications.

\section{Introduction}

Prostate cancer ( $\mathrm{PCa}$ ) ranks as the second prevalent cause of death among men. In the past twenty years, prostate specific antigen has been regarded as the most frequently used and important biomarker for screening, diagnosis, staging and prognosis of PCa [1]. The PSA is produced by the prostate to liquefy the seminal fluids, which is a glycoprotein and circulates in serum by two different molecular morphology: uncomplexed PSA-a1-antichymotrypsin complex (free PSA, fPSA) and complexed PSA (cPSA). The fPSA accouts for $10 \%-30 \%$ of total PSA (tPSA) and other PSA lack immunological activity. For the diagnosis of PCa, the criterion for biomarker of PSA as following: the value of tPSA above 10 $\mathrm{ng} / \mathrm{ml}$ is evaluated as the positive, indicating high risk of PCa; The value of tPSA below $4 \mathrm{ng} / \mathrm{ml}$ is regarded as the negative and low possibility; the value in the range of $4 \mathrm{ng} / \mathrm{ml}$ to $10 \mathrm{ng} / \mathrm{ml}$ is so-called "grey zone" and the ration of fPSA/tPSA affects the precise diagnosis of PCa.

A variety of techniques have been developed for the determination of fPSA/tPSA including the electrochemical impedimetric immunosensors [2], bead-array fluorescence assay [3] and screen-printed electrochemical biosensor [4]. However above-mentioned immunoassay have suffered from several shortcomings, such as limited sensitivity, sophisticated procedures/operation and hard to miniaturization. Over the past years, organic electrochemical transistors (OECTs) have attracted intensive attentions for various biosensing applications including ions, [5, 6] dopamine [7], cells [8], bacteria [9], pH 
[11-14]. The OECTs translate the small signal of ionic fluxes into an amplified electrical readout. As a typical OECTs, a wildly accepted semiconducting poly (3, 4- ethylenedioxythiophene): poly (styrenesulfonate) (PEDOT: PSS) would assist three terminal electrodes (gate, source and drain) to transducer biochemical signal with high gain. In particularly, the gain performance could be evaluated by following equation:

$G_{\mathrm{m}}=\frac{\mathrm{Wd}}{\mathrm{L}} \mu C^{*}\left(V_{\mathrm{th}}-V_{\mathrm{G}}\right)($ Eq. 1)

where $C^{*}$ is the volumetric capacitance, and $V_{\text {th }}$ is the threshold voltage.

From above relationship, the structure of OECTs and the mobility decide its performance [15-17]. Previous researchers have reported interdigitated structure to improve the ratio of $W / L$ without significantly increasing device area, which validated the feasibility of interdigitated architecture for amplification of OECTs biosensors [18, 19]. Meanwhile, electrodes with multiple spiral have been reported for biosensing applications due to the unique structure [20,21]. However, the OECTs with interdigitated multiple spiral hasn't reported for the biosensing of fPSA/tPSA.

Transition metal carbide or nitride (MXene) has been regarded as next-generation two-dimensional (2D) nanomaterials for wide biomedical applications, including biosensing, photocatalysis, supercapacitor and lithium-ion battery, because of its unique characteristics [22-25]. Two-dimensional $\mathrm{Ti}_{3} \mathrm{C}_{2} \mathrm{Tx}$ (MXene) has been recently explored for biosensing, such as glucose [26], hemoglobin [27], gliotoxin [28] and carcinoembryonic antigen (CEA) [29]. Considering the intercalation and delamination of layered carbides and carbonitrides, MXene nanocomposites provide an ideal site for structural rearrangement semiconducting nanomaterials [30-32]. However, there are no reports for combination of MXene and PEDOT: PSS for synergistic determination of fPSA/tPSA.

Herein, in this paper, we proposed a dual OECTs biosensor with optimized electrode architecture and MXene-assisted semiconducting nanomaterials for the ultrasensitive quantification of fPSA/tPSA. Moreover, our assay afforded the enhanced detection limit of fPSA and tPSA protein down to $0.01 \mathrm{pg} / \mathrm{ml}$ $(S / N>3)$, as well as satisfactory selectivity, reproducibility and reliability, comparable to the best previous literatures. Finally, the clinical performance of the dual OECTs biosensor were evaluated against commercial device and demonstrated comparable linear linearity and high correlation coefficient in biosample quantification.

\section{Materials And Methods}

\section{Materials}

Prostate specific antigen, cluster of differentiation 44 (CD44), cluster of differentiation 63 (CD63), carcinoembryonic antigen (CEA), alpha-fetoprotein (AFP) were purchased from Abcam, Inc. (Cambridge, MA). Monoclonal anti-Free PSA (ab1. PSA30) and monoclonal anti-Total PSA (ab2, PSA10) were provide Loading [MathJax]/jax/output/CommonHTML/jax.js 
by Guan Xian ShenglangsaiBio-Technology Co., Ltd (Chins). The PEDOT: PSS (PH 1000) aqueous solutions was purchased from Heraeus Clevios GmbH (Germany, Leverkusen). The 3-Mercaptopropionic acid (MCA), d-sorbitol, ethylene glycol, BSA (bovine serum albumin), glucose, lysine, tyrosine, (3Glycidyloxypropyl) trimethoxysilane (GOPS), potassium ferricyanide $\left(\mathrm{K}_{3}\left[\mathrm{Fe}(\mathrm{CN})_{6}\right]\right)$ and potassium ferrocyanide $\left(\mathrm{K}_{4}\left[\mathrm{Fe}(\mathrm{CN})_{6}\right]\right)$, MAX phase $\mathrm{Tl}_{3} \mathrm{ALC}_{2}$, hydrochloric acid $(\mathrm{HCL})$ and lithium fluoride (LiF) were purchase from Sigma Chemical Co (St. Louis, MO). Purified deionized (DI) water was obtained by a MilliQ system (M $\left.\Omega \cdot \mathrm{cm} @ 25^{\circ} \mathrm{C}\right)$. The photoresist AZ5214 was purchased from MicroChem (USA). The phosphate buffered saline (PBS) and all other chemicals were purchased from Aladdin Chemicals Co., Ltd.

\section{Synthesis of MXene}

The $\mathrm{Ti}_{3} \mathrm{C}_{2}$ nanosheets was successfully prepared by etching $\mathrm{Al}$ from $\mathrm{Ti}_{3} \mathrm{AlC}_{2}$ in $\mathrm{HF}$ according to reported literatures $[22,33,34]$. Original MAX phase was ball milled for $8 \mathrm{~h}$ with the weight ratio of 10:1 (speed of $450 \mathrm{RPM}$ ), and then the $\mathrm{Tl}_{3} \mathrm{AlC}_{2}$ was filtered by sieve. $\mathrm{Ti}_{3} \mathrm{C}_{2}$ was synthesized by selectively etching the Allayer from $\mathrm{Ti}_{3} \mathrm{AlC}_{2}$ in $\mathrm{LiF} / \mathrm{HCl}$ systems. Typically, $2 \mathrm{~g}$ of $\mathrm{LiF}$ was dissolved in $50 \mathrm{~mL}$ of $\mathrm{HCl}$ under magnetic stirring for $15 \mathrm{~min}$. Then $3 \mathrm{~g}$ of processed $\mathrm{Ti}_{3} \mathrm{AlC}_{2}$ powered slowly added into the mixture and heated at $45^{\circ} \mathrm{C}$ for $10 \mathrm{~h}$ with stirring. The obtained solution was repeatedly washed three times with deionized water until of $\mathrm{pH}$ of $\sim 6.5$. Finally the resulting supernatant was collected and stored at $4{ }^{\circ} \mathrm{C}$ for the subsequent usage.

\section{Preparations of MXene@PEDOT: PSS nanocomposites}

To realize the transistor channels, we prepared a mixture of PEDOT: PSS with d-sorbitol (40\% by volume), $1 \%$ GOPS (by volume, as a crosslinker), 0.1\% DBSA (by volume, for the improvement of film processing and wettability) and DMSO (5\% by volume). Then, the MXene@PEDOT: PSS nanocomposites were prepared by dropwise incubation of colloidal MXene into the mixture of PEDOT: PSS. Finally, the mixture was filtered by nanoporous polypropylene membranes (Millipore $0.22 \mu \mathrm{m}, 47 \mathrm{~mm}$, Nylon) in air and stored at $4{ }^{\circ} \mathrm{C}$ for the subsequent usage.

\section{Fabrication of isMOECTs biosensor}

The fabrication of biosensor included the ultraviolet lithography, deposition of metal (thermal evaporation technique) and spin-coating of MXene@PEDOT: PSS nanocomposites. Before the ultraviolet lithography, the native oxide $\mathrm{p}^{+}$silicon substrates were cleaned with acetone, isopropanol and water. Then the silicon substrate were properly dried and exposed $\mathrm{O}_{2}$ plasma for 3 mins. Subsequently, a customized shadow mask with interdigitated multiple spiral was prosposed. After that, the $p+$ silicon substrate was photolithographically patterned by photoresist AZ5214 (MicroChem) with a positive tone lift-off process. For the deposition of metal layer, the patterned Cr/Au source, drain electrodes (thicknesses: $6 \mathrm{~nm} / 80 \mathrm{~nm}$ ) were thermally evaporated evaporation on substrates. Consequently, the mixture of PEDOT: PSS@MXene nanocomposites was spin-coated and anneal at $150{ }^{\circ} \mathrm{C}$ in nitrogen environment for 2 hr. Finally, the devices were washed with deionized water. blow dried with nitrogen gun before use and stored at $4{ }^{\circ} \mathrm{C}$. 


\section{Apparatus}

Characterizations techniques such as scanning electron microscopy (SEM), contact angle, atomic force microscopy (AFM), electrochemical impedance spectroscopy (EIS) and. differential pulse voltammetry (DPV) were carried out to analyzed the properties of the materials and sensors. The SEM characterization was performed by Hitachi S-4800 by $10000 x$ magnifications at an accelerating voltage $12 \mathrm{kV}$. The Data physics SZ-CAMA1 was used to analyzed the contact angle, where $10 \mu \mathrm{L}$ of purified water droped on the isMOECTs biosensor for observations. The morphological characterization of isMOECTs biosensors was conducted by Dimension Icon \& FastScan Bio (Bruker Nano, California, USA) with resonance frequency range of $100-400 \mathrm{kHz}$. The transfer characteristics of biosensor isMOECTs biosensor was measured by Keithley 2612B (Tektronix Co Ltd.).

The electrochemical analysis were carried out on an electrochemical workstation $(\mathrm{CHI660e}, \mathrm{CH}$ Instrument, Shanghai). For the DPV electrochemical measurement, the scanning voltage was ranged from 0 to $450 \mathrm{mV}$ with a pulse amplitude of $20 \mathrm{mV}$, a pulse width of $30 \mathrm{~ms}$, and a quiet time of $2 \mathrm{~s}$. For the EIS characterization, the frequency was performed in the range of $0.1-20000 \mathrm{~Hz}$ with a pulse amplitude of $10 \mathrm{mV}$.

For the parameters and settings of isMOECTs measurements, the source, drain and gate electrodes of the OECTs were linked to two channels sourcemeter instrument (Keithley 2612B, and a customizable MATLAB program was designed to modulate gate voltages $\left(\mathrm{V}_{\mathrm{G}}\right)$ and source-drain voltages $\left(\mathrm{V}_{\mathrm{DS}}\right)$ and record the changes of data.

To characterize the responses of isMOECTs various concentrations of fPSA/tPSA protein, transfer characteristics of isMOECTs was selected to under following parameters: IDS was tested at a fixed sourcedrain voltage $\left(V_{D S}=0.08 \mathrm{~V}\right)$ at various gate voltages $\left(V_{G}: 0 \sim 1.1 \mathrm{~V}\right)$ with a sweeping rate of $0.02 \mathrm{Vs}^{-1}$. For the parameters of output characteristics $\left(\mathrm{I}_{\mathrm{DS}} \mathrm{VS} \mathrm{V}_{\mathrm{D}}\right)$, the $\mathrm{V}_{\mathrm{G}}$ was fixed at $0.9 \mathrm{~V}$ at various drain voltages $\left(\mathrm{V}_{\mathrm{D}}: 0-0.2 \mathrm{~V}\right)$ with a scanning rate of $0.01 \mathrm{~V} / \mathrm{s}$.

\section{Harvesting of biosamples and statistical analysis}

The Institutional Ethics Committee of the Pudong New Area People's Hospital affiliated to Shanghai University of Medicine and Health Sciences approved all clinical biosamples and protocols in this project (No: PRYLZ 2018-039-A). For the procedures of biosamples harvesting, biosamples ( $5 \mathrm{~mL}$ ) were centrifuged at $1500 \times \mathrm{g}$ for $10 \mathrm{~min}$ and the residue supernatant was obtained in heparin tube (green top) and stored at $-80^{\circ} \mathrm{C}$ for further determination. All calibration plot, standard deviation (S.D.), T-Test and relative standard deviation (RSD) were analyzed by the SPSS software (version 17.0, SPSS Statistics, Inc: Chicago USA). The receiver operating characteristics curve was analyzed by Graphpad Prism (V9.1.2.226). 


\section{Preparation of MXene@PEDOT: PSS and design of isMOECTs}

As schematically illustrated in Fig. 1, we performed the synthesis procedures of MXene@PEDOT: PSS semiconductor membrane and the fabrication of isMOECTs biosensor. The Fig. 1a illustrated the key components of isMOECTs biosensor, including six-channel ZIF connector, dual organic electrochemical transistors with respective interdigitated spiral-amplifier architecture. Figure $1 \mathrm{~b}$ was the equivalent circuit between the channel of isMOECTs and the gate electrode. For the synthesis of MXene@PEDOT: PSS, the crucial element is the synthesis of micropatterned MXene with intercalation and delamination of layered architecture and subsequent structural rearrangement with PEDOT: PSS, which is significant for amplification of amperometric signal of isMOECTs biosensor. As demonstrated in Fig. 1c, the MXene and PEDOT: PSS were structurally arranged into MXene@PEDOT: PSS nanocomposites under continuous stirring, while the PEDOT: PSS recombined with the intercalation/delamination layer of MXene. We conducted the scanning electron microscopy (SEM) of MXene@ PEDOT: PSS by the front view (Fig. 1d) and vertical view (Fig. 1e) to validate the morphological and structural arrangement of MXene @PEDOT: PSS and pure MXene (Fig. S1). Another key characteristics of isMOECTs biosensor for ultrasensitive determination of fPSA/tPSA is interdigital multiple spiral, which displayed in Fig. $1 \mathrm{f}$ with different number of spiral for amperometric-signal amplification.

\section{Functionalization of isMOECTs}

For the specificity of isMOECTs biosensor, we performed fPSA/tPSA functionalizations on the dual gate electrodes in Fig. 2a. We performed the contact angles, cyclic voltammetry (CV), electrochemical impedance and atomic force microscopy (AFM) to characterize the modification processes. For the contact angle (Fig. 2b), the value decreased from $68.35^{\circ}$ (blue, Fig. 2b) to $65.43^{\circ}$ (black, Fig. 2b) and $47.17^{\circ}$ (red, Fig. $2 \mathrm{~b}$ ), respectively, after modification of MCA and anti-tPSA, due to the high hydrophilicity of MCA and anti-tPSA. For the cyclic voltammetry, the peak current declined from $85.31 \mu \mathrm{A}$ (blue curve in Fig. 2c) to $66.12 \mu \mathrm{A}$ (black curve in Fig. 2c) and $55.41 \mu \mathrm{A}$ (red curve in Fig. 2c), respectively. We also conducted the investigation of scan rate-dependency study (Fig. S2), revealing a linear behavior $\left(I_{\text {redox }}=\right.$ $\left.31.171 \times \mathrm{V}^{1 / 2}-18.031, \mathrm{R}^{2}=0.9947\right)$ between the electrochemical current and the scan rate $(\mathrm{V})$. For the EIS (Fig. 2d), the $R_{\text {et }}$ demonstrated $385.4 \Omega$ (blue curve), $812.3 \Omega$ (black curve) and $1038.4 \Omega$ (red curve) for $\mathrm{Au}, \mathrm{Au} / \mathrm{COOH}, \mathrm{Au} / \mathrm{COOH} /$ anti-tPSA. The trend of $\mathrm{CV}$ and EIS analysis could be attributed to the blocking of electron via MCA and anti-tPSA. We also conducted the AFM to observe the morphological changes in Fig. S3. The root mean square (rms) roughness represents the morphological characteristics of isMOECTs biosensor, including the bare gate electrode $(7.8 \pm 1.9 \mathrm{~nm}), \mathrm{Au} / \mathrm{MCA}(46.3 \pm 8.5 \mathrm{~nm})$, $\mathrm{Au} / \mathrm{MCA} /$ anti-tPSA $(135.3 \pm 19.5 \mathrm{~nm})$, which validated functionalization procedures. Abovementioned characterizations (contact angle, CV, EIS and AFM) demonstrated successful modification of isMOECTs biosensor for the specific quantifications of fPSA/tPSA. 
To achieve ultrasensitive determination performance of isMOECTs biosensor, we optimized the ratio of MXene/PEDOT: PSS and the architecture of interdigitated multiple spiral. As show in Fig. S4, we performed the ratio of in 2:1, 1:1, 1.5:1 and 2:1, and the ratio of 1:1 demonstrated highest transconductance in the transfer characteristics. For the optimization of spiral architecture, we selected the number of $6,12,18$, and the number of 12 exhibited highest amperometric amplification of isMOECTs biosensor with the maximum transconductance of $0.99 \mathrm{mS}$ (Fig. S5-6). Moreover, we also optimized parameters among the concentration of antibody $(20 \mu \mathrm{g} / \mathrm{mL}), \mathrm{pH}$ value of 7.0 , temperature of $37^{\circ} \mathrm{C}$ and incubation of 40 mins for optimum configurations of isMOECTs (Fig. S7). Based on above optimizations. We performed typical quantifications of fPSA/tPSA as Fig. 3a with corresponding transfer characteristics and transconductance in Fig. 3b. Subsequently, as shown in Fig. 3c-d, we recorded the sensitive determination of fPSA with wide linear wide of $10 \mathrm{fg} / \mathrm{mL}-100 \mathrm{ng} / \mathrm{mL}$ (the detection limit of $5.33 \mathrm{fg} / \mathrm{mL}$ ), and the corresponding linear regression curve was $\Delta V_{g s}=7.63 \times \log \left(C_{f p s a}\right)-6.69, R^{2}=0.992$ with the slope of $8.89 \mathrm{mV} / \mathrm{dev}$. The detection limit of each device was defined by the channel current response at a signal/noise ratio of $3[35,36]$. For the purpose of comparisons, the typical gate electrode with antifPSA functionalization was selected as the working electrode by differential pulse voltammetry (DPV) technique to detect fPSA. As demonstrated in Fig. 3e-f, the determination performance of DPV technique achieved the detection range of $1000 \mathrm{fg} / \mathrm{mL}-100 \mathrm{ng} / \mathrm{mL}$, with the detection limit of $432.5 \mathrm{fg} / \mathrm{mL}$. The tPSA quantification performance of isMOECTs and its corresponding DPV results were also illustrated in Fig. S8. Our purposed isMOECTs demonstrated higher sensitivity than conventional electrochemical methods and other type biosensors (such as electrochemical impedimetric immunosensors, bead-based fluorescence assay and reflectance spectroscopy) with summarized comparisons in Supporting Information, Table S1, which could be attributed to the combination of micropatterned MXene@PEDOT: PSS and interdigitated multiple spiral architecture.

\section{Selectivity and reproducibility}

We selected three high-weight molecules and two small-molecules as interferences to evaluate the selectivity of isMOECTs biosensor. As illustrated in Fig. 4a, the anti-fPSA-functionalized isMOECTs biosensor displayed high specificity for quantification of $10 \mathrm{ng} / \mathrm{mL}$ fPSA with the value of $16.8 \pm 0.43 \mu \mathrm{A}$, which was obviously high than $10 \mathrm{ng} / \mathrm{mL}$ tPSA $(0.51 \pm 0.21 \mu \mathrm{A}), 100 \mathrm{ng} / \mathrm{mL} \mathrm{CD} 44(0.31 \pm 0.11 \mu \mathrm{A}), 100$ $\mathrm{ng} / \mathrm{mL}$ AFP $(0.33 \pm 0.15 \mu \mathrm{A}), 100 \mathrm{ng} / \mathrm{mL}$ CA125 $(0.69 \pm 0.24 \mu \mathrm{A}), 100 \mathrm{ng} / \mathrm{mL}$ glucose $(0.42 \pm 0.13 \mu \mathrm{A}), 100$ $\mathrm{ng} / \mathrm{mL}$ tyrosine $(0.33 \pm 0.12 \mu \mathrm{A})$ and blank $(0.11 \pm 0.04 \mu \mathrm{A})$, demonstrating high specificity of isMOECTs biosensor for quantification of fPSA. Moreover, anti-fPSA-functionalized isMOECTs biosensor displayed high specificity for quantification of tPSA against other interferences (Fig. 4b).

For the reproducibility, we recorded amperometric signals of seven anti-fPSA-functionalized isMOECTs biosensors and seven anti-tPSA-functionalized isMOECTs biosensors with RSD values of $1.19 \%$ and $1.51 \%$, respectively, demonstrating acceptable reproducibility of proposed isMOECTs biosensors (Fig. 4cd). For the stability, compared with original status, the amperometric signal exhibited negligible variation with decline by < $5 \%$ (Fig. 4e-f), affording applicable stability of proposed isMOECTs biosensor. 


\section{Clinical performance}

In clinics, the isMOECTs biosensors were subsequently applied in a cohort of 49 human serum (Supporting Information, Table S2), and the biosamples were standardly harvested according typical protocol (in Experiment section). As displayed in Fig. 5a-b, and isMOECTs biosensor achieved high correlation with referenced value for the quantification of PPSA and IPSA, respectively. Moreover, according to the statistical analysis of TPSA/fPSA via isMOECTs biosensors, we calculated all ratio value of fPSA/tPSA in Fig. $5 c$ with $p<0.01$. Finally, we performed the receiver operating characteristic (ROC) analysis in Fig. $5 \mathrm{~d}$. The isMOECTs biosensors afforded good sensitivity $(75.8 \%)$ and specificity $(75.0 \%)$, as well as excellent area under the curve (AUC) value of 0.8138 (confidence interval: $95 \%$ ) against referenced clinical chemiluminescence immunoassay (Beckham DXI600), confirming the potential applications of isMOECTs in clinics.

\section{Conclusions}

In summary, isMOECTs biosensor was used in this work as a potential diagnostic tool for sensitive and specific quantification of fPSA/tPSA in prostate cancer patients. By combination of the micropatterned MXene@PEDOT: PSS and multiple spiral architecture, the isMOECTs biosensor afforded improved assay detection sensitivity and range as well as high specificity, reproducibility and reliability. Moreover, the isMOECTs displayed excellent area under the curve (AUC) value of 0.8138 , confirming the potential applications of isMOECTs in clinics. This is first demonstration of MXene-based spiral-amplifier organic electrochemical transistor biosensor for human (prostate) cancer diagnosis and our strategy would be extended to detect other biomarkers in various types of cancer or for liquid biopsy, paving a convenient and versatile platform for quantification of cancer-relevant biomarkers.

\section{Declarations}

\section{Acknowledgement}

No applicable.

\section{Author' contributions}

SWX and JJS conceived the idea and designed the experiments. YCZ performed the experiments. BC, QJ, YZ and YCZ analyzed the data. SWX, JJS and YCZ wrote and revised the manuscript. All authors read and approved the final manuscript.

\section{Funding}

This study was supported by the Outstanding Young Medical Talent Training Program of Pudong Health Bureau of Shanghai (PWRq2020-40) and National Natural Science Foundation of China (81901747). 
All data generated or analyzed during this study are included in this article.

\section{Ethics approval and consent to participate}

Not applicable.

\section{Consent for publication}

All authors are Consent for publication.

\section{Competing interests}

The authors declare that they have no known competing financial interests.

\section{Author details}

aDepartment of Ultrasound, Pudong New Area People's Hospital affiliated to Shanghai University of Medicine and Health Sciences, Shanghai 201200, China;

${ }^{\text {b}}$ Department of Urology, Renji Hospital, School of Medicine, Shanghai Jiao Tong University, Shanghai 200127, China;

'Department of Ultrasound, Renji Hospital, School of Medicine, Shanghai Jiao Tong University, Shanghai 200127, China.

*Corresponding author: Prof Shaowei Xie, Tel: 0086-21-68383396; E-mail: xieshaowei@renji.com. Prof Jianjun Sha, Tel: 0086-21-68383396; E-mail: shajianjunuro@163.com.

\section{References}

1. Consortium MFGAPPCAS (GAP3), Bruinsma SM, Roobol MJ, Carroll PR, Klotz L, Pickles T, et al. Semantics in active surveillance for men with localized prostate cancer-results of a modified Delphi consensus procedure. Nat Rev Urol. 2017;14:312-22.

2. Han L, Wang D, Yan L, Petrenko VA, Liu A. Specific phages-based electrochemical impedimetric immunosensors for label-free and ultrasensitive detection of dual prostate-specific antigens. Sensors Actuators, B Chem. 2019;297.

3. Cao D, Li CY, Qi CB, Chen HL, Pang DW, Tang HW. Multiple optical trapping assisted bead-array based fluorescence assay of free and total prostate-specific antigen in serum. Sensors Actuators B Chem. 2018;269:143-50.

4. Escamilla-Gómez V, Hernández-Santos D, González-García MB, Pingarrón-Carrazón JM, Costa-García A. Simultaneous detection of free and total prostate specific antigen on a screen-printed electrochemical dual sensor. Biosens Bioelectron. 2009;24:2678-83. 
5. Sessolo M, Rivnay J, Bandiello E, Malliaras GG, Bolink HJ. lon-selective organic electrochemical transistors. Adv Mater. 2014;26:4803-7.

6. Lin P, Yan F, Chan HLW. Ion-sensitive properties of organic electrochemical transistors. ACS Appl Mater Interfaces. 2010;2:1637-41.

7. Tang H, Lin P, Chan HLW, Yan F. Highly sensitive dopamine biosensors based on organic electrochemical transistors. Biosens Bioelectron. 2011;26:4559-63.

8. Lingstedt LV, Ghittorelli M, Brückner M, Reinholz J, Crăciun NI, Torricelli F, et al. Monitoring of Cell Layer Integrity with a Current-Driven Organic Electrochemical Transistor. Adv Healthc Mater. 2019;8.

9. Méhes G, Roy A, Strakosas X, Berggren M, Stavrinidou E, Simon DT. Organic Microbial Electrochemical Transistor Monitoring Extracellular Electron Transfer. Adv Sci. 2020;7:2000641.

10. Inal S, Rivnay J, Suiu AO, Malliaras GG, McCulloch I. Conjugated Polymers in Bioelectronics. Acc Chem Res. 2018;51:1368-76.

11. Mariani F, Gualandi I, Tessarolo M, Fraboni B, Scavetta E. PEDOT: Dye-Based, Flexible Organic Electrochemical Transistor for Highly Sensitive pH Monitoring. ACS Appl Mater Interfaces. 2018;10:22474-84.

12. Wustoni S, Combe C, Ohayon D, Akhtar MH, McCulloch I, Inal S. Membrane-Free Detection of Metal Cations with an Organic Electrochemical Transistor. Adv Funct Mater. 2019;29:1-10.

13. Spyropoulos GD, Gelinas JN, Khodagholy D. Internal ion-gated organic electrochemical transistor: A building block for integrated bioelectronics. Sci Adv. 2020;5.

14. Fu Y, Wang N, Yang A, Law HK wai, Li L, Yan F. Highly Sensitive Detection of Protein Biomarkers with Organic Electrochemical Transistors. Adv Mater. 2017;29:1-7.

15. Savagian LR, Österholm AM, Ponder JF, Barth KJ, Rivnay J, Reynolds JR. Balancing Charge Storage and Mobility in an Oligo(Ether) Functionalized Dioxythiophene Copolymer for Organic- and AqueousBased Electrochemical Devices and Transistors. Adv Mater. 2018;30.

16. Donahue MJ, Williamson A, Strakosas X, Friedlein JT, McLeod RR, Gleskova H, et al. HighPerformance Vertical Organic Electrochemical Transistors. Adv Mater. 2018;30.

17. Paterson AF, Faber H, Savva A, Nikiforidis G, Gedda M, Hidalgo TC, et al. On the Role of Contact Resistance and Electrode Modification in Organic Electrochemical Transistors. Adv Mater. 2019;31.

18. Liang Y, Brings F, Maybeck V, Ingebrandt S, Wolfrum B, Pich A, et al. Tuning Channel Architecture of Interdigitated Organic Electrochemical Transistors for Recording the Action Potentials of Electrogenic Cells. Adv Funct Mater. 2019;29:1-10.

19. Liang Y, Wu C, Figueroa-Miranda G, Offenhäusser A, Mayer D. Amplification of aptamer sensor signals by four orders of magnitude via interdigitated organic electrochemical transistors. Biosens Bioelectron. 2019;144.

20. Sardesai AU, Dhamu VN, Paul A, Muthukumar S, Prasad S. Design and Electrochemical Characterization of Spiral Electrochemical Notification Coupled Electrode (SENCE) Platform for Biosensina Application. Micromachines. 2020;11.

Loading [MathJax]/jax/output/CommonHTML/jax.js 
21. Hou Y, Zhang R, Luo H, Liu G, Kim Y, Yu S, et al. Microbial electrolysis cell with spiral wound electrode for wastewater treatment and methane production. Process Biochem. 2015;50:1103-9.

22. Xu B, Zhu M, Zhang W, Zhen X, Pei Z, Xue Q, et al. Ultrathin MXene-Micropattern-Based Field-Effect Transistor for Probing Neural Activity. Adv Mater. 2016;28:3333-9.

23. Wang QW, Zhang H, Bin, Liu J, Zhao S, Xie X, Liu L, et al. Multifunctional and Water-Resistant MXeneDecorated Polyester Textiles with Outstanding Electromagnetic Interference Shielding and Joule Heating Performances. Adv Funct Mater. 2019;29.

24. Shang M, Chen X, Li B, Niu J. A Fast Charge/Discharge and Wide-Temperature Battery with a Germanium Oxide Layer on a Ti3C2 MXene Matrix as Anode. ACS Nano. 2020;14:3678-86.

25. Wang F, Yang C, Duan M, Tang Y, Zhu J. TiO2 nanoparticle modified organ-like Ti3C2 MXene nanocomposite encapsulating hemoglobin for a mediator-free biosensor with excellent performances. Biosens Bioelectron England. 2015;74:1022-8.

26. Lei Y, Zhao W, Zhang Y, Jiang Q, He J, Baeumner AJ, et al. A MXene-based wearable biosensor system for high-performance in vitro perspiration analysis. Small Wiley Online Library. 2019;15:1901190.

27. Liu H, Duan C, Yang C, Shen W, Wang F, Zhu Z. A novel nitrite biosensor based on the direct electrochemistry of hemoglobin immobilized on MXene-Ti3C2. Sensors Actuators B Chem. 2015;218:60-6.

28. Wang H, Li H, Huang Y, Xiong M, Wang F, Li C. A label-free electrochemical biosensor for highly sensitive detection of gliotoxin based on DNA nanostructure/MXene nanocomplexes. Biosens Bioelectron. 2019;142:111531.

29. Wu Q, Li N, Wang Y, Xu Y, Wei S, Wu J, et al. A 2D transition metal carbide MXene-based SPR biosensor for ultrasensitive carcinoembryonic antigen detection. Biosens Bioelectron. 2019;144:111697.

30. Qin L, Tao Q, El Ghazaly A, Fernandez-Rodriguez J, Persson POÅ, Rosen J, et al. High-Performance Ultrathin Flexible Solid-State Supercapacitors Based on Solution Processable Mo1.33C MXene and PEDOT:PSS. Adv Funct Mater. 2018;28.

31. Bai S, Guo X, Chen T, Zhang Y, Zhang X, Yang H, et al. Solution processed fabrication of silver nanowire-MXene@PEDOT: PSS flexible transparent electrodes for flexible organic light-emitting diodes. Compos Part A Appl Sci Manuf. 2020;139:106088.

32. Mashtalir O, Naguib M, Mochalin VN, Dall'Agnese Y, Heon M, Barsoum MW, et al. Intercalation and delamination of layered carbides and carbonitrides. Nat Commun. 2013;4.

33. Zhou T, Wu C, Wang Y, Tomsia AP, Li M, Saiz E, et al. Super-tough MXene-functionalized graphene sheets. Nat Commun [Internet]. Springer US; 2020;11:1-11. Available from: http://dx.doi.org/10.1038/s41467-020-15991-6.

34. Chen WY, Jiang X, Lai SN, Peroulis D, Stanciu L. Nanohybrids of a MXene and transition metal dichalcogenide for selective detection of volatile organic compounds. Nat Commun [Internet].

Loading [MathJax]/jax/output/CommonHTML/jax.js om: http://dx.doi.org/10.1038/s41467-020-15092-4. 
35. Lavagnini I, Antiochia R, Magno F. A calibration-base method for the evaluation of the detection limit of an electrochemical biosensor. Electroanalysis. 2007;19:1227-30.

36. Park SJ, Kwon OS, Lee SH, Song HS, Park TH, Jang J. Ultrasensitive flexible graphene based fieldeffect transistor (FET)-type bioelectronic nose. Nano Lett. 2012;12:5082-90.

\section{Figures}
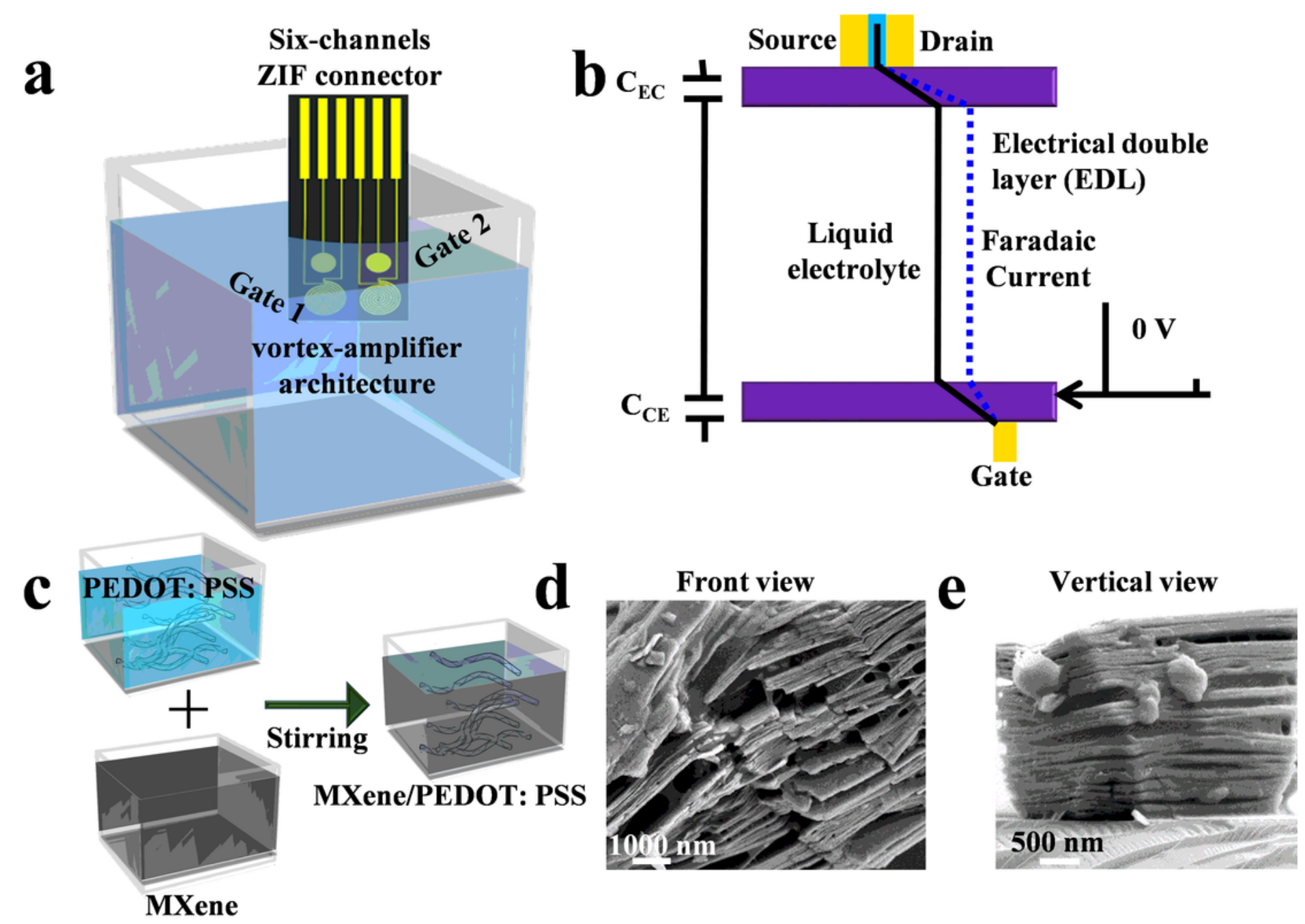

f

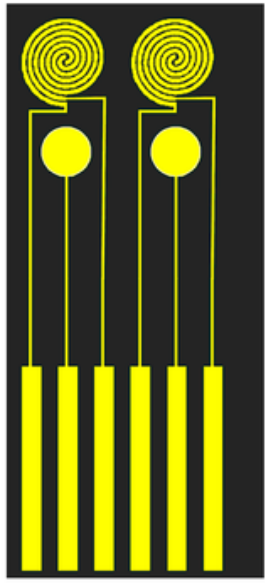

$\mathrm{N}=6$

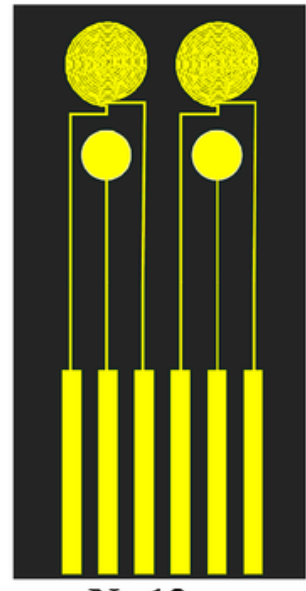

$\mathbf{N}=12$

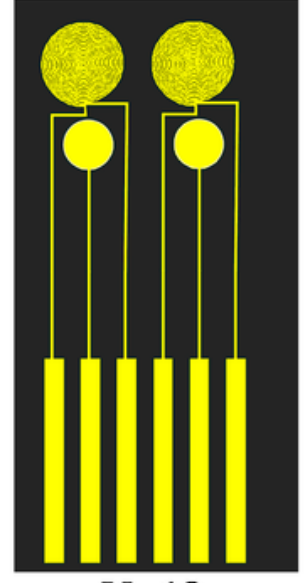

$\mathbf{N}=18$ 
Figure 1

Illustrative schematic of isMOECTs biosensors for quantification of fPSA/tPSA. a) Typical configurations of isMOECTs biosensor, and b) corresponding equivalent circuit. c) The preparation procedures for MXene@PEDOT: PSS nanocomposites.SEM characterizations of MXene@PEDOT: PSS via d) front view and e) vertical view. $f$ ) The configurations of isMOECTs biosensors with different number of interdigitated spirals.

a

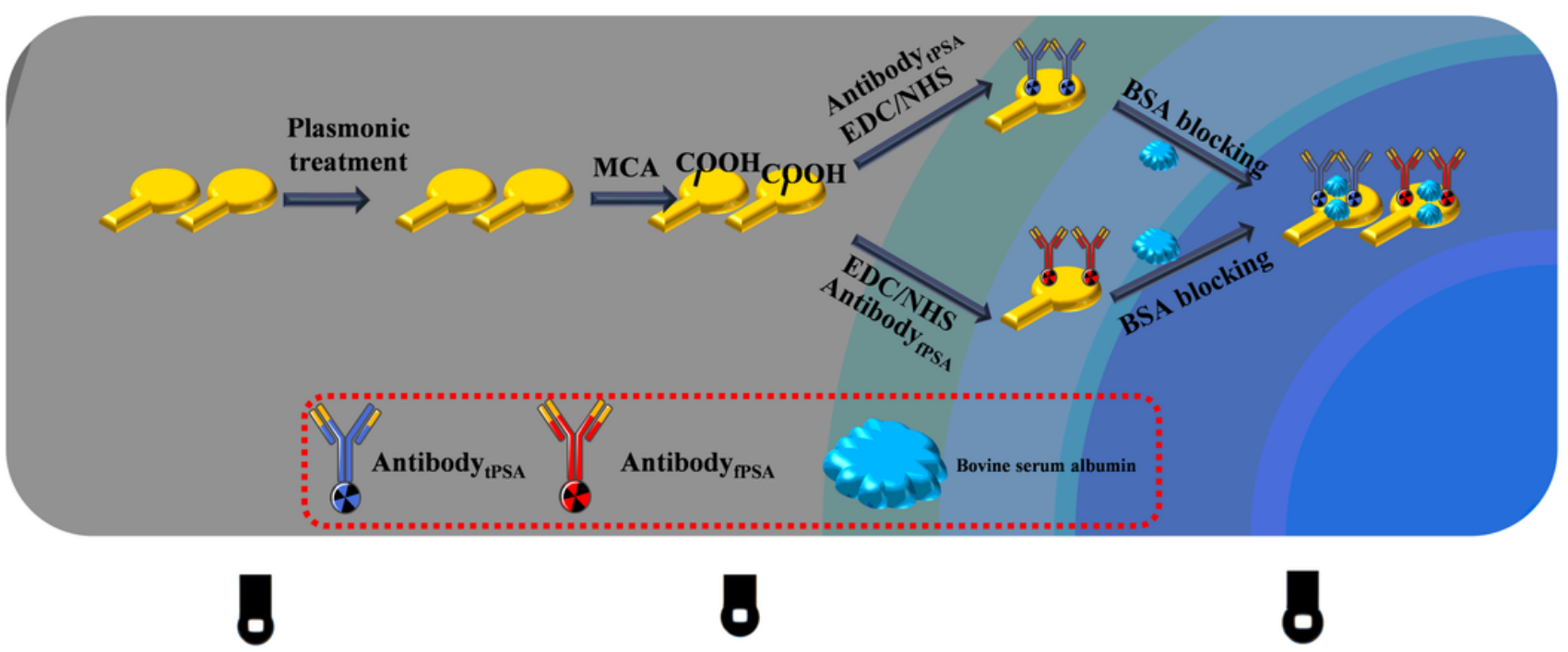

b

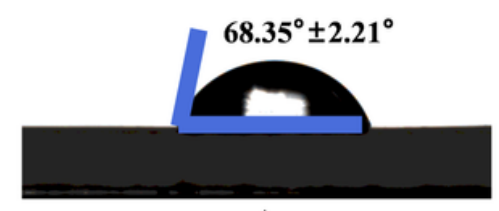

Au

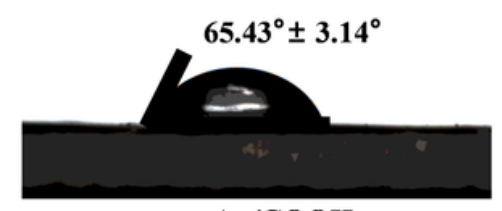

$\mathrm{Au} / \mathrm{COOH}$

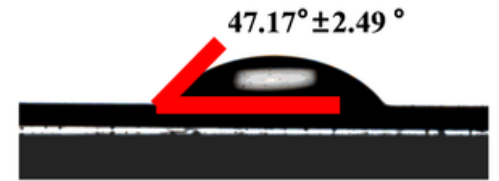

Au/COOH/antibody tPSA $_{\text {A }}$ c

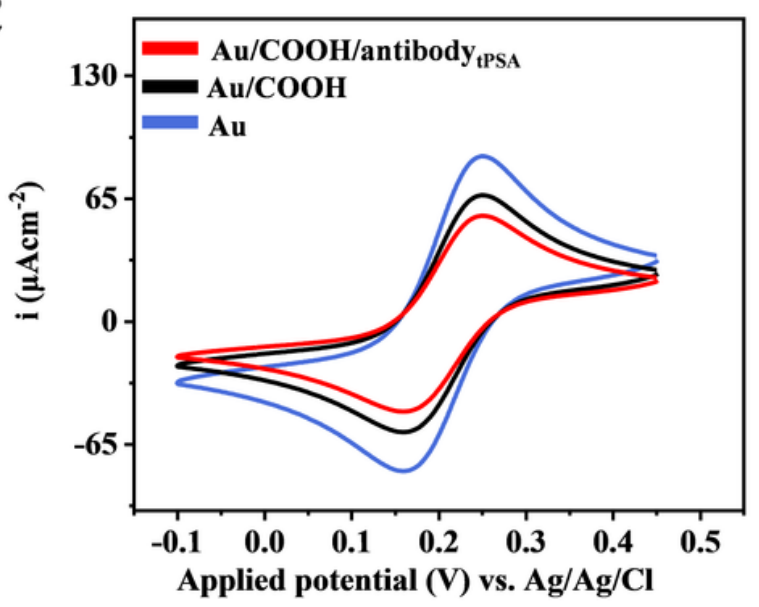

d

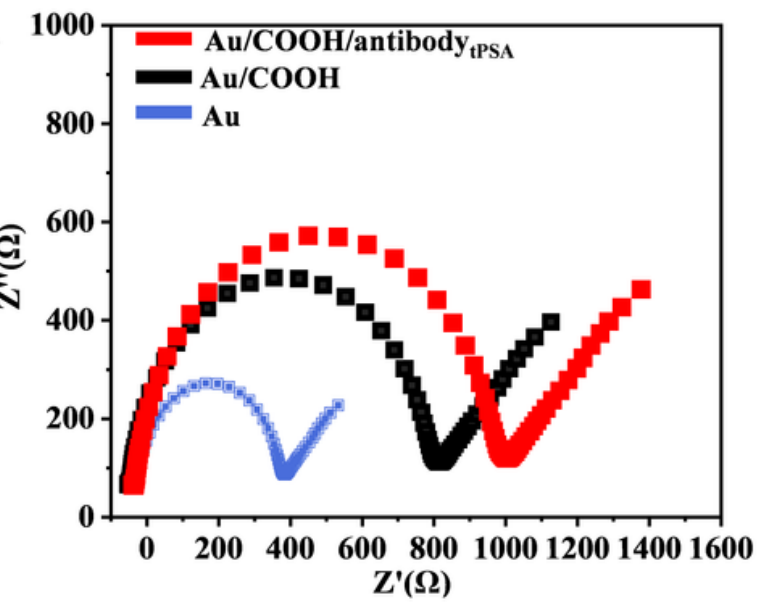

Figure 2 
Representations of layer-by-layer functionalizations and characterizations of gate electrode on isMOECTs biosensor. a) The modification procedures, b) EIS, c) CV and d) contact angle analysis for the gate electrode.
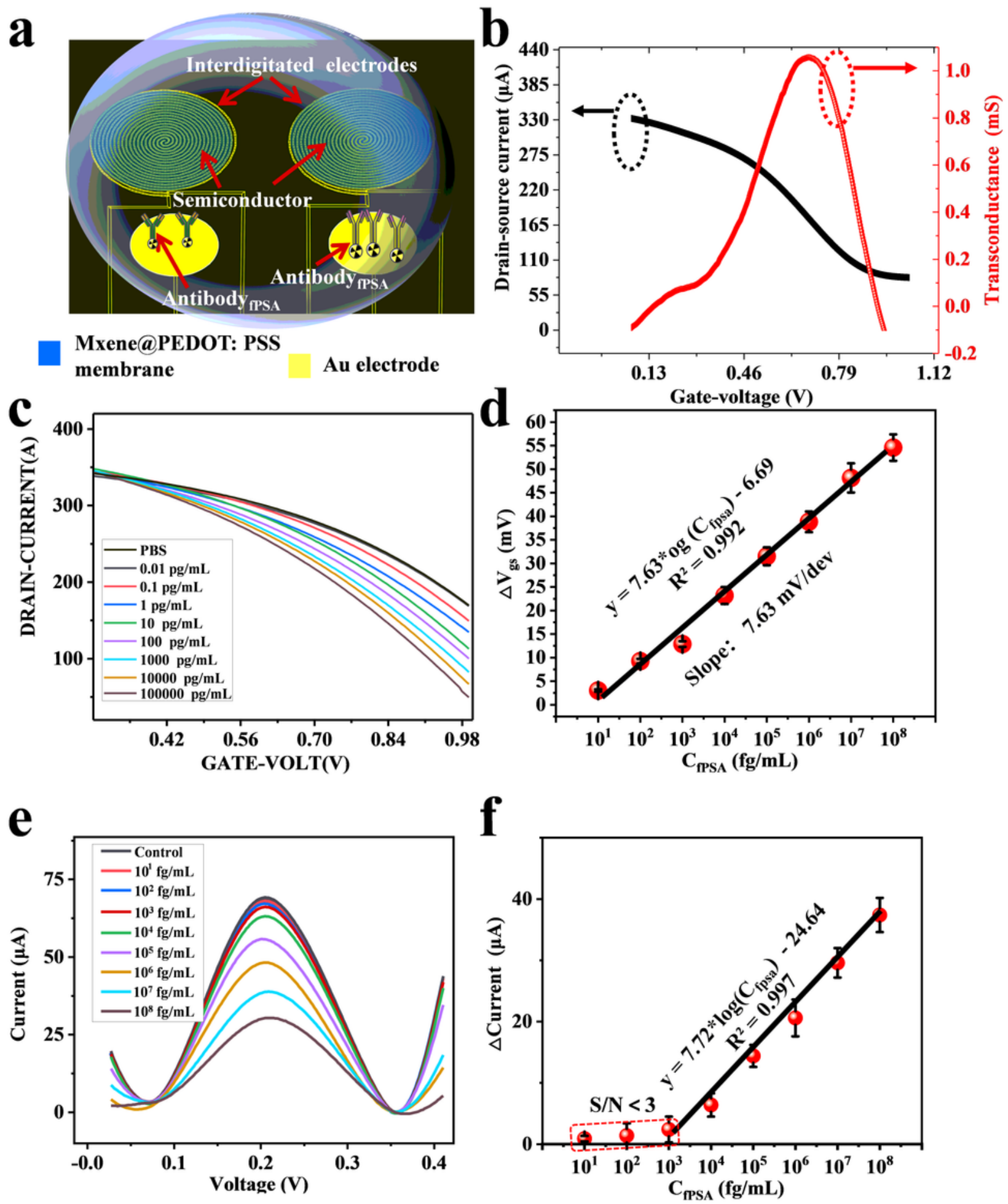

Figure 3

Depiction of quantification mechanism and performance. a) The conceptual mechanism of isMOECTs Loading [MathJax]/jax/output/CommonHTML/jax.js ypical transfer characteristics with transconductance. c) The 
transfer characteristics response of the isMOECTs biosensor to the addition of fPSA, d) drain current as a function of fPSA. e) Traditional DPV measurement of the Au gate electrode as the working electrode in the incubation of a series of fpsa. f) The calibration plot of the DPV testing as a function of fPSA. Each of error in panels $d$ and $f$ is three replicated analysis $(n=3)$.
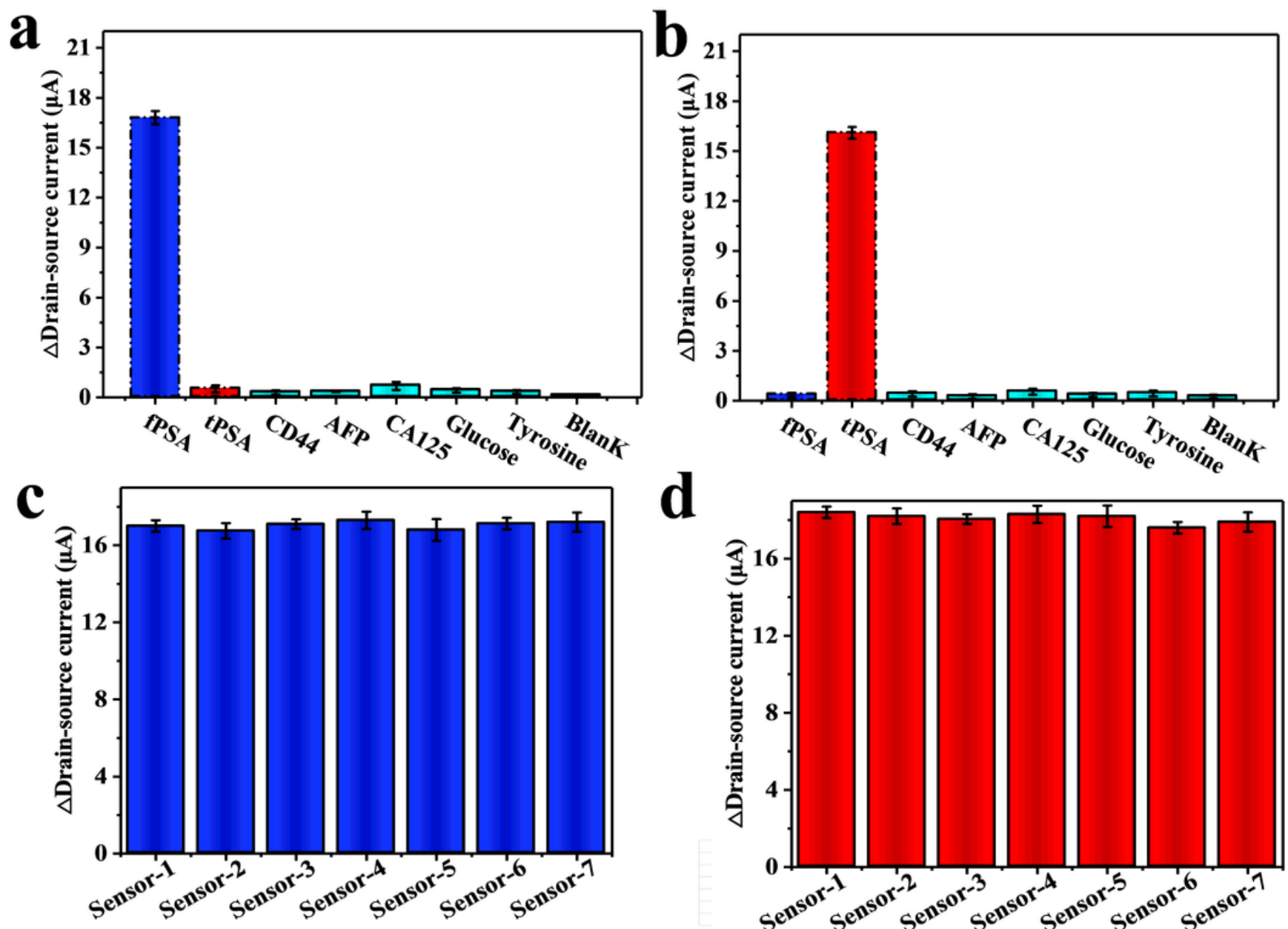

d
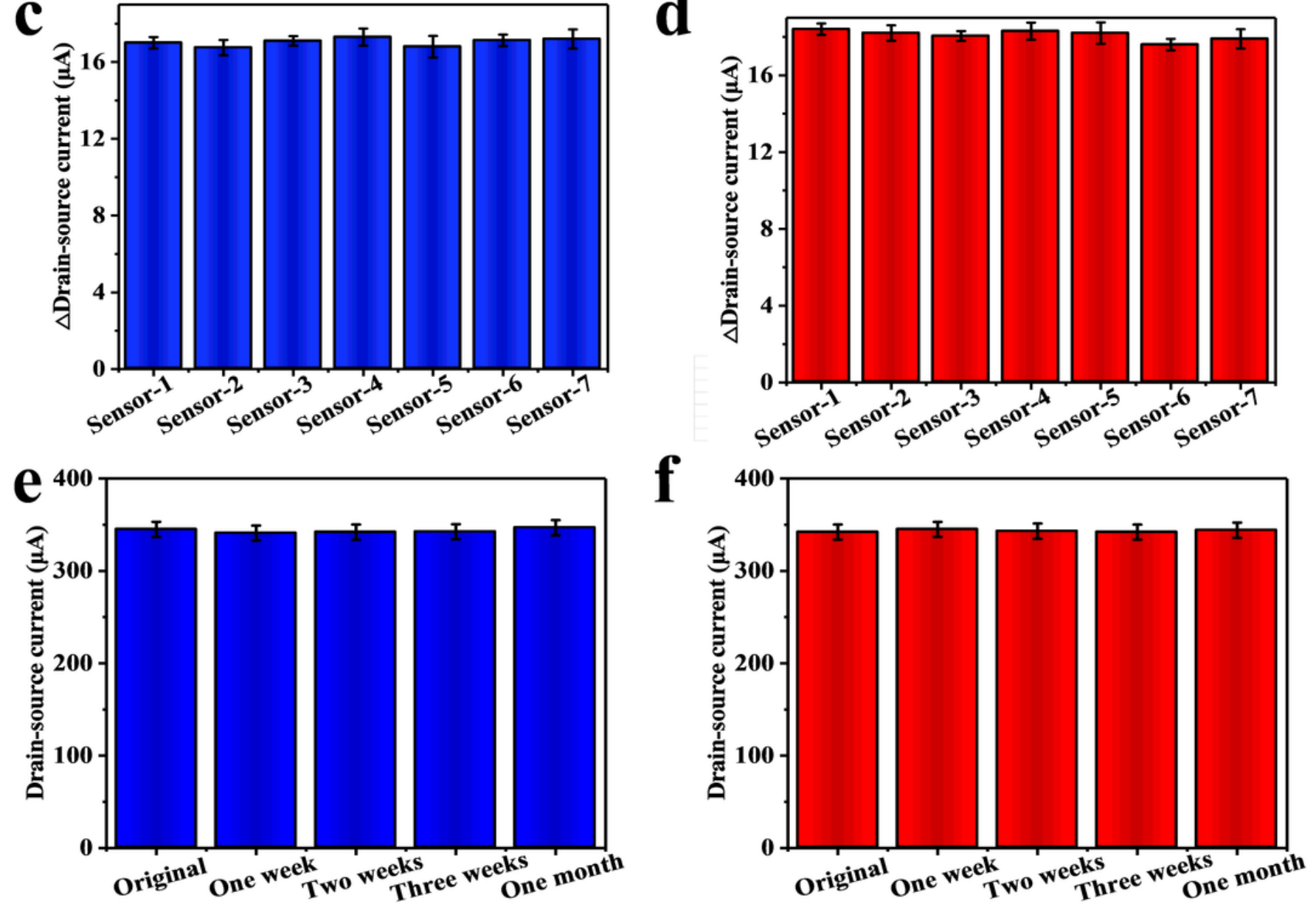

\section{Figure 4}

Performance evaluations. a) The selectivity, c) reproducibility and e) stability assessment of isMOECTs Loading [MathJax]/jax/output/CommonHTML/jax.js e selectivity, d) reproducibility and e) stability assessment of 
isMOECTs biosensors for determination of tPSA. Each of error in panels a-e is three replicated analysis $(n=3)$
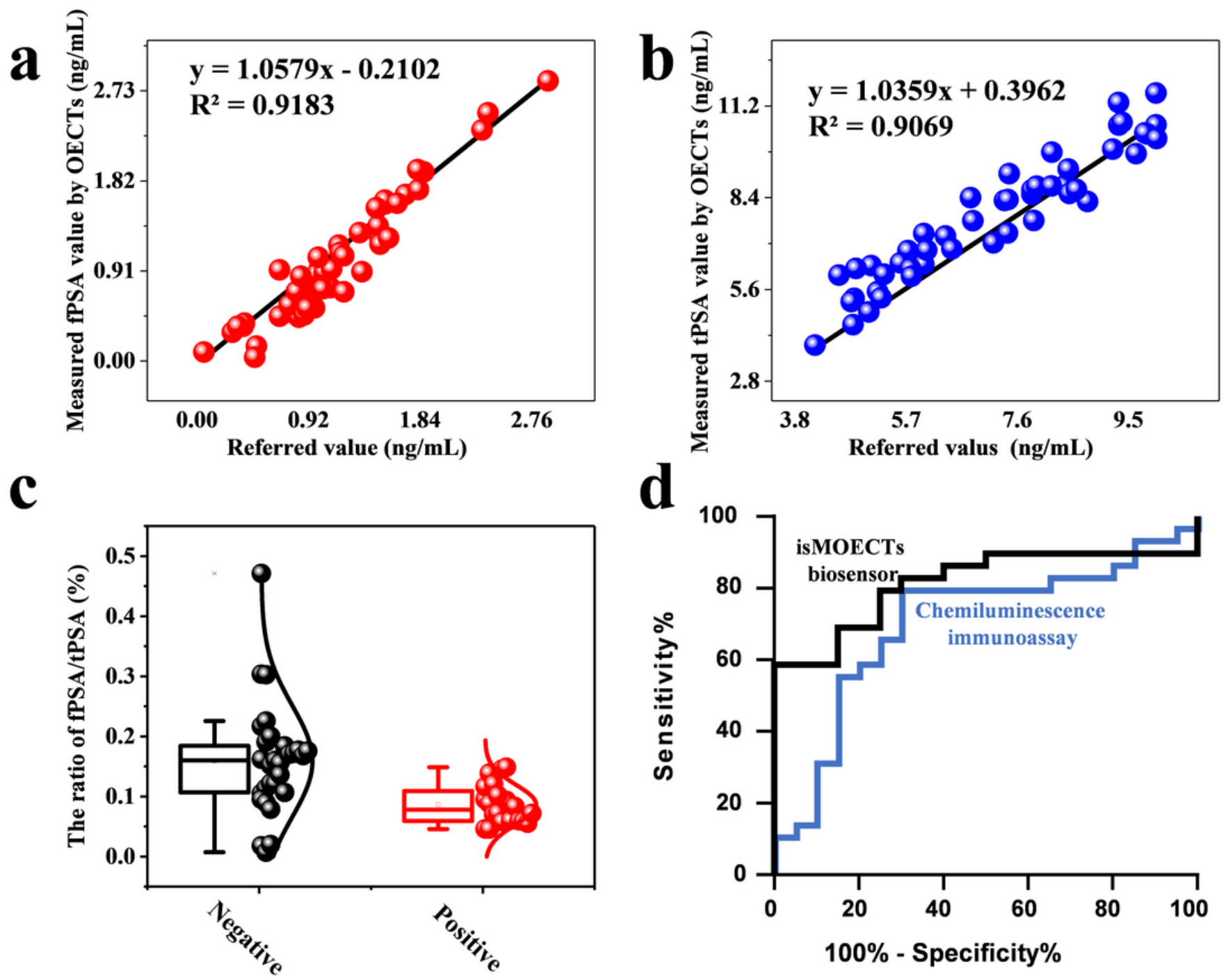

Figure 5

Clinics assessments for 49 patient biosamples. a) Correlation analysis between reference values (Beckham DXI600) and measured value (via isMOECTs) for quantification of a) fPSA and b) tPSA. c) The T-test discrimination for the ratio of fPSA/tPSA among all biosamples. d) The comparisons of receiver operating characteristic between the chemiluminescence immunoassay (blue curve, Beckham DXI600) and the isMOECTs biosensors (black curve).

\section{Supplementary Files}

This is a list of supplementary files associated with this preprint. Click to download.

- Graphicalabstractimage.jpg 